\title{
Electrophysiologic actions and antifibrillatory efficacy of subacute left stellectomy in a conscious, post-infarction canine model of ischemic ventricular fibrillation
}

\author{
Steven D. Nelson, Joseph J. Lynch, David Sanders, \\ Daniel G. Montgomery and Benedict R. Lucchesi \\ Departments of Pharmacology and Internal Medicine (Division of Cardiologw. The University of Michigan Medical School. \\ Ann Arbor, Michigan. U.S.A.
}

(Received 28 December 1987; revision accepted 25 August 1988)

\begin{abstract}
Nelson SD, Lynch JJ, Sanders D, Montgomery DG, Lucchesi BR. Electrophysiologic actions and antifibrillatory efficacy of subacute left stellectomy in a conscious, post-infarction canine model of ischemic ventricular fibrillation. Int J Cardiol 1989;22:365-376.
\end{abstract}

The autonomic nervous system appears to modulate ventricular arrhythmias associated with acute myocardial ischemia. This study investigated the electrophysiologic effects and antifibrillatory actions of subacute left stellectomy in a conscious, post-infarction canine model of sudden cardiac death.

Twenty-two dogs with a previous anterior wall myocardial infarction and inducible ventricular arrhythmias were randomized to undergo either left stellectomy $(n=12)$ or remain as sham-denervated controls $(n=10)$. Five to 7 days post left stellectomy, there were no significant changes in heart rate, electrocardiographic intervals or ventricular refractoriness compared to sham-denervated controls. Acute posterolateral ischemia was produced in left stellectomy and sham-denervated dogs by anodal current-induced thrombosis via a previously positioned electrode in the left circumflex coronary artery. Ventricular fibrillation developed within 1 hour of the onset of ischemia (early ventricular fibrillation) in 3/12 (25\%) left stellectomy dogs versus $8 / 10(80 \%)$ sham-denervated controls $(P<0.05)$. However, 24 -hour mortality rate was $5 / 12(42 \%)$ after left stellectomy versus $8 / 10(80 \%)$ after sham denervation $(P=0.072)$. Small differences in regional myocardial norepinephrine content, which is a marker for neuronal integrity, occurred in the mid-posterolateral and mid-anteroseptal regions of the left ventricle after left stellectomy. Overall norepinephrine concentration after left stellectomy was $409.70 \pm 9.90 \mathrm{ng} / \mathrm{g}$ vs $428.07 \pm 10.84 \mathrm{ng} / \mathrm{g}$ in sham controls ( $P=$ NS).

In summary, subacute left stellectomy significantly reduces the incidence of ventricular fibrillation occurring within 1 hour of the onset of acute posterolateral ischemia at a distance to a previous myocardial infarction in conscious dogs, and tends to reduce the ischemic post-infarction mortality at 24 hours after the

Correspondence to: Steven D. Nelson, M.D., Division of Cardiology. The Ohio State University Hospitals, 663 Means Hall, 1654 Upham Drive, Columbus, OH 43210, U.S.A.

Supported by the National Institutes of Health. Heart, Lung and Blood Institutes Grant No. HL-05806-27. Dr. Nelson was a Fellow of the American Heart Association of Michigan during the conduct of this study. 
onset of ischemia. This protective effect of left stellectomy is not due to any alteration in cardiac electrophysiologic parameters measured prior to the development of acute posterolateral ischemia, nor is it related to regional denervation as determined by myocardial tissue concentration of residual norepinephrine.

Key words: Ventricular arrhythmias; Myocardial ischemia; Autonomic nervous system

\section{Introduction}

The sympathetic nervous system is reported to play an important role in the genesis of malignant ventricular arrhythmias [1-4]. Several experimental studies in open chest, anesthetized animal models have demonstrated that left stellate ganglion stimulation can reduce ventricular refractoriness, reduce the ventricular fibrillation threshold and may produce ventricular arrhythmias $[5,6]$. In contrast, acute left stellate ganglionectomy reduces the incidence of ventricular arrhythmia associated with transient coronary occlusion [7], can increase ventricular fibrillation threshold [8] and prolong the ventricular refractory period [9]. More recently, Schwartz et al. have demonstrated an antifibrillatory effect of chronic left stellectomy in conscious post-infarction dogs $[10,11]$. To date, no study has assessed the relationship between the electrophysiologic effects of left stellectomy and how they might relate to the antifibrillatory actions of left stellectomy in a conscious animal preparation.

The series of experiments presented in this investigation attempt to expand upon the previous observations made by Schwartz et al. $[10,11]$. The electrophysiologic actions and antiarrhythmic efficacy of subacute, rather than chronic, left stellectomy are assessed in a conscious post-infarction canine model of ischemic ventricular fibrillation. This study addresses whether the actions of subacute left stellectomy are related to regional myocardial denervation as assessed by depletion of myocardial catecholamine stores. The conscious post-infarction dog model used in the present evaluation has been shown to display a strong positive correlation between susceptibility to programmed ventricular stimulation-induced ventricular tachycardia and vulnerability to subsequent ischemic ventricular fibrillation, thus permitting the identification of post-infarction preparations at "high risk" toward lethal ischemic arrhythmias for this study [12]. This preparation has been used extensively by this laboratory for the characterization and identification of potentially efficacious antifibrillatory interventions in the post-infarction setting [13].

\section{Methods}

Male mongrel dogs weighing $15-20 \mathrm{~kg}$ were anesthetized with sodium pentobarbital $(30 \mathrm{mg} / \mathrm{kg}$ i.v.), intubated and artifically ventilated with a Harvard respirator. Using aseptic technique, the left internal jugular and the left common carotid were isolated and cannulae were inserted. The

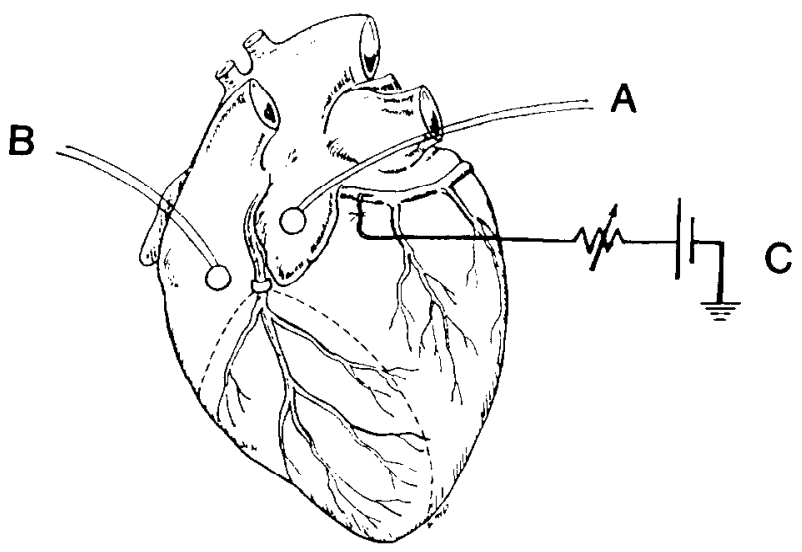

Fig. 1. Surgical preparation of the conscious canine model of sudden death. An anterior wall myocardial infarction (broken line) is produced by a 2-hour occlusion of the left anterior descending artery, followed by reperfusion through a critical stenosis. An atrial epicardial bipolar electrode (A) is sutured onto the left atrial appendage for atrial pacing. A bipolar plunge electrode $(B)$ is inserted into the interventricular septum near the right ventricular outflow tract for determination of ventricular excitation threshold, ventricular refractory period and for the delivery of extra stimuli during programmed ventricular stimulation. A bared tip silver wire (C) is inserted into the lumen of the proximal left circumflex coronary artery and secured (see text). 
cannulae were passed subcutaneously to the back of the neck and exited through a small stab wound. Then a thoracotomy was performed in the left fourth intercostal space using blunt dissection. 'The heart was suspended in a pericardial cradle (Fig. 1). The mid left anterior descending artery was isolated and partially occluded with a critical stenosis as previously described [12,14]. The artery then was occluded using a snare formed from a loop of silastic tubing passed through a polyethylene tube. Perfusion through the left anterior descending coronary artery was restored after 2 hours of occlusion. A bipolar pacing electrode $(1 \mathrm{~mm}$ diameter silver wire, $3 \mathrm{~mm}$ apart) was sutured to the surface of the left atrial appendage. A bipolar plunge electrode ( 25 gauge insulated stainless steel wire, $5 \mathrm{~mm}$ in length, $2 \mathrm{~mm}$ apart) was secured in the interventricular septum at the right ventricular outflow tract. The proximal left circumflex coronary artery was isolated using blunt dissection. 'The bared tip of a 30 gauge leflon coated silver wire was inserted into the lumen of the left circumflex coronary artery. The wire then was secured to the epicardium with 3 sutures. Then the left stellate ganglion, ansa subclavia and the rami communicantes from $\mathrm{T} 1$ to $\mathrm{T} 4$ of the sympathetic chain were identified. Using a technique described by Schwartz and Stone [10], suture snares were positioned carefully around the caudal portion of the ganglion near the point where $\mathrm{T} 2$ ramus enters the sympathetic chain and around the ansa subclavia at the cranial portion of the ganglion. All wires and stellate sutures were exteriorized carefully. The chest was closed in layers. Silver disc electrodes then were implanted subcutaneously for electrocardiographic monitoring. The surgical incisions were closed and the animals allowed to convalesce. Pre- and post-operative animal care was in strict accordance with "The Guide for the Care and Use of Laboratory Animals", DEW pub. No. (NIH) 78-23.

\section{Experimental protocol}

Dogs were returned to the laboratory 3 to 5 days after anterior myocardial infarction. Electrophysiologic testing and ventricular programmed electrical stimulation were performed while the animals were conscious and resting comfortably in a sling. Only those dogs that were susceptible to the initiation of either nonsustained or sustained ventricular tachycardia at baseline programmed stimulation testing were entered into the present investigation. Previous work in this laboratory has demonstrated that post-infarction dogs that have inducible nonsustained or sustained ventricular tachycardia at baseline testing are at "high risk" toward the development of lethal ventricular arrhythmias in response to acute posterolateral ischemia (i.e., ischemia at a site remote from previous myocardial infarction) [14].

Immediately after initial electrophysiologic testing, post-infarction dogs were randomized to undergo either left stellate ganglionectomy or to remain as sham-denervated controls. Left stellectomy was performed during sedation with thiamylal sodium ( $6 \mathrm{mg} / \mathrm{kg}$ i.v.), by acutely extirpating the suture snares previously positioned around the left stellate ganglion. Evidence for effective left stellectomy was the presence of part or all of the stellate ganglion on the extirpated sutures and the immediate development of miosis, ptosis and elevation of the nictitating membrane in the left eye due to loss of left sympathetic tone. Visual confirmation of left stellectomy was performed at autopsy. In the sham-denervated controls, the sutures were not extirpated.

Five to 7 days post left stellectomy or after a comparable period of time in sham-denervated controls, all post-infarction dogs were subjected to repeat electrophysiologic testing in the conscious state. Immediately thereafter, sham-denervated controls $(n=10)$ and left stellectomy dogs $(n=12)$ were entered into the protocol for left circumflex intimal injury and posterolateral ischemia.

\section{Electrophysiologic study}

Animals were studied while they were conscious and resting comfortably in a sling. ECG intervals and electrophysiologic parameters were determined immediately before programmed ventricular stimulation. ECG intervals, including a rate-corrected $\mathrm{QT}$ interval ( $\mathrm{QTc}=\mathrm{QT}$ in $\mathrm{msec}$ ) $(\mathrm{R}-\mathrm{R} \text { in sec })^{1 / 2}$ ) were measured in sinus rhythm. $A$ paced $Q T$ interval was measured during atrial 
pacing at a drive cycle length of $400 \mathrm{msec}$. Right ventricular outflow tract excitation threshold and refractory period were measured also during an atrial pacing drive cycle length of $400 \mathrm{msec}$. The right ventricular outflow tract excitation threshold was the minimum voltage required to produce a conducted ventricular impulse $\left(V_{2}\right)$ using a single ventricular extrastimulus $\left(S_{2}\right)$ with $4 \mathrm{msec}$ pulse duration delivered $300 \mathrm{msec}$ after the $\mathrm{R}$ wave of the lead II ECG. The right ventricular outflow tract refractory period was the longest $\mathrm{R}-\mathrm{S}_{2}$ interval at which a twice threshold voltage stimulus of 4 msec pulse duration failed to elicit a $V_{2}$ response.

Programmed electrical stimulation was performed using a Grass Model S- 88 stimulator and a Grass model SIU-5 stimulation isolation unit. The pacing protocol was identical to that used by this laboratory in previous investigations $[12,14]$. Briefly, premature ventricular extrastimuli (4 msec duration, $2 \times$ threshold) were introduced into the interventricular septum via the right ventricular outflow tract bipolar electrode during sinus rhythm. Single $\left(\mathrm{S}_{2}\right)$, double $\left(\mathrm{S}_{2} \mathrm{~S}_{3}\right)$ and the triple $\left(\mathrm{S}_{2} \mathrm{~S}_{3} \mathrm{~S}_{4}\right)$ extrastimuli were entered at progressively shorter coupling intervals until ventricular tachycardia ( 5 or more repetitive ventricular beats) was induced reproducibly or until a minimum coupling interval of $125 \mathrm{msec}$ was achieved. This protocol has been shown previously not to induce ventricular arrhythmias in non-infarction dogs [14].

Acute posterolateral ischemia in the setting of a previous anterior wall myocardial infarction: a model for ischemic ventricular fibrillation

After post-treatment electrophysiology studies, an anodal direct current of $150 \mu \mathrm{A}$ was applied to the intimal surface of the left circumflex coronary artery via the previously positioned, intraluminal silver electrode. The anodal current induces intimal damage, vasomotion, thrombosis and ultimately ischemia in the posterolateral wall of the left ventricle. Lead II of the surface ECG was monitored continuously by a Grass Polygraph or recorded at preset intervals by a programmable cardio-cassette recorder throughout the experiment. Left circumflex artery current was con-

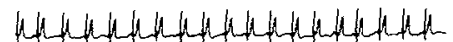

0 MIN

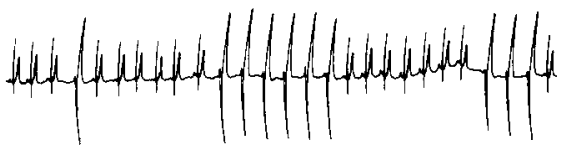

210 MIN

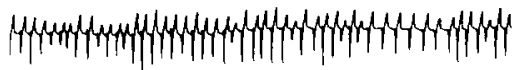

24 HOURS

Fig. 2. Example of electrocardiographic monitoring of lead II in a conscious post-infarction dog with left stellectomy (LSGx) which survived acute posterolateral ischemia. A continuous anodal current of $150 \mu \mathrm{A}$ was applied to the intimal surface of the left circumflex coronary artery at time 0 . At $210 \mathrm{~min}$ of current, electrocardiographic evidence of premature ventricular depolarizations and ST-T wave changes suggestive of acute myocardial ischemia or injury are present. At 24 hours of current, the dog is alive with $100 \%$ ventricular ectopy.

tinued for 24 hours (Fig. 2) or until ventricular fibrillation developed (Fig. 3). Upon completion of the experiment, the chest was opened and the
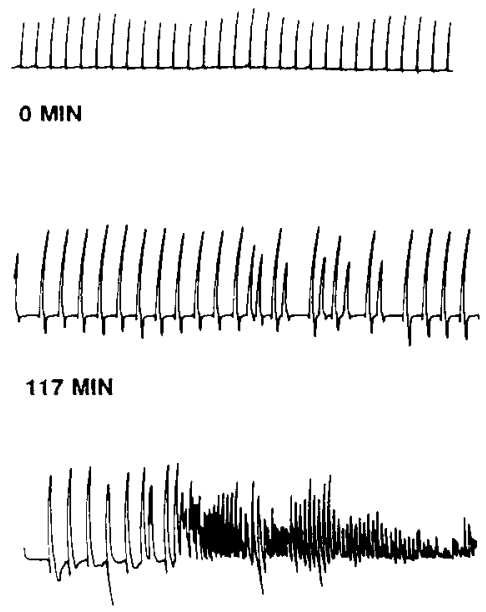

$122 \operatorname{MIN}$

Fig. 3. Example of lead Il electrocardiographic monitoring in a conscious post-infarction dog without left stellectomy (LSGx), which did not survive acute posterolateral ischemia. Again. a continuous $150 \mu \mathrm{A}$ current is applied to the intimal surface of the left circumflex coronary artery at time 0 . At time $117 \mathrm{~min}$ there is electrocardiographic evidence of an idioventricular rhythm with premature ventricular depolarizations suggestive of acute ischemia or injury. At $122 \mathrm{~min}$ ventricular fibrillation develops spontaneously. 
left paravertebral region was exposed to visually inspect stellate destruction and then the heart was excised. The left circumflex coronary artery was isolated carefully, and the intravascular thrombus was removed and weighed. The heart was cut into $1 \mathrm{~cm}$ thick transverse sections which were incubated in $0.5 \%$ triphenyltetrazolium chloride in $0.01 \mathrm{M}$ phosphate buffer ( $\mathrm{pH} 7.4)$. Reaction with triphenyltetrazolium forms a red precipitate in viable tissue, while infarcted tissue remains pale $[15,16]$. Size of infarction was quantitated gravimetrically, and was expressed as a percentage of total left ventricle.

\section{Evaluation of myocardial norepinephrine con- centrations after subacute left stellectomy}

Norepinephrine concentrations were determined in ventricular samples from 10 dogs $(5$ post left stellectomy and 5 sham-denervated controls) in order to evaluate the effect of left stellate ganglion ablation on myocardial norepinephrine content. The left stellate ganglion was isolated via a left thoracotomy as described above. During surgery, the left stellate ganglia in one group of dogs were extirpated by the placement of and subsequent pulling of the sutures around the ganglia. In the second group, sutures were placed loosely around the ganglia, but were not otherwise manipulated. One week post left stellectomy, dogs were anesthetized with Dial-Urethane solution (10\% allobarbital, $40 \%$ monoethyl urea, $40 \%$ urethane; $0.6 \mathrm{ml} / \mathrm{kg}$ i.v.) and were ventilated as described above. After a repeat left thoracotomy, the hearts were excised during supramaximal stimulation of the right vagus nerve (4 $\mathrm{msec}$ pulse duration, $40 \mathrm{~Hz}, 1.5 \times$ threshold voltage) to cause sinus arrest, thereby minimizing the efflux of catecholamines from tissue stores during this procedure.

After excision of the heart, transverse sections of tissue were made rapidly along the apex-base axis. A transverse section of the heart from the mid left ventricle containing prominent anterior and posterior papillary muscles which could be used as orientation markers was chosen for tissue sampling. From this section, transmural ventricular myocardial samples of approximately $1 \mathrm{~g}$ each were labelled and frozen rapidly in liquid nitrogen after excision from the following regions of the ventricle: (1) anterior, (2) anterolateral, (3) posterolateral, (4) posterior, (5) posteroseptal, and (6) anteroseptal. Frozen tissue samples were weighed and then placed individually in $0.3 \mathrm{~N}$ perchloric acid (10 ml of perchloric acid/1.0 g of tissue) for thawing and for homogenization using a tissue homogenizer. The homogenate was centrifuged for $20 \mathrm{~min}$ at $20,000 \times \mathrm{g}\left(4^{\circ} \mathrm{C}\right)$. Catecholamine concentrations were determined in the supernatant using high pressure liquid chromatography with an electrochemical detector according to the method of Goldstein and co-workers [17]. The limit of sensitivity for this assay was $5.0 \mathrm{pg}$ per sample. The assay was performed in the Diabetes Research and Training Center Ligand Laboratory at The University of Michigan Medical Center.

\section{Effects of acute left stellectomy in post infarction, anesthetized, open chest dogs}

This experiment was designed to verify that the technique of left stellectomy employed in this investigation (i.e. neural transection at the level of the ansa subclavia and at the junction of the $T 2$ ramus) [10] would produce a functional interruption of left-sided efferent sympathetic input to the heart.

A separate group of male mongrel dogs $(n=5)$ with 3- to 5-day-old anterior myocardial infarctions produced by techniques described above, were re-anesthetized, intubated and ventilated. A repeat thoracotomy was performed and the heart was suspended in a pericardial cradle. A Walton Brodie strain gauge was sutured to the noninfarcted posterolateral wall of the left ventricle to measure left ventricular contractility. The left femoral artery was isolated and cannulated for continuous monitoring of arterial pressure. Lead II of the surface ECG was monitored. The left stellate ganglion was isolated. Bipolar pacing electrodes were positioned on the left sympathetic chain just caudal to the T2 ramus. Heart rate, mean arterial pressure and isometric force of contraction were measured at baseline and during incremental sympathetic chain stimulation $(1,2,4$ then $8 \mathrm{~Hz}, 2$ msec pulse width) both before and 30 
to $60 \mathrm{~min}$ after the left stellate was sectioned at the level of the ansa subclavia and at the junction of the T2 ramus. The preparations also were challenged with tyramine $(25 \mu \mathrm{g} / \mathrm{kg}$, i.v. $)$ pre and post stellectomy.

\section{Statistical analysis}

For all evaluations, data are expressed as mean \pm SEM. For the electrophysiology studies, preand post-treatment values were compared, when appropriate, by a two-tailed Student's paired (within group) or unpaired (between groups) $t$-test. Regional norepinephrine concentrations were compared between left stellectomy and sham controls by the Student's unpaired $t$-test. Differences in survival were analyzed by a Fisher's Exact test. A $P$ value $<0.05$ was the criterion for statistical significance.

\section{Results}

Electrophysiologic effects of subacute left stellectomy in conscious, post-infarction dogs

Twenty-two post-infarction dogs underwent electrophysiologic testing both before and 5-7 days post left stellectomy $(n=12)$ or sham denervation $(n=10)$. The electrocardiographic and electrophysiologic effects of left stellectomy are summarized in Table 1 Both QTc and paced QT intervals were shortened significantly after left stellectomy $(292 \pm 9$ vs $262 \pm 6 \mathrm{msec} ; P<0.01)$

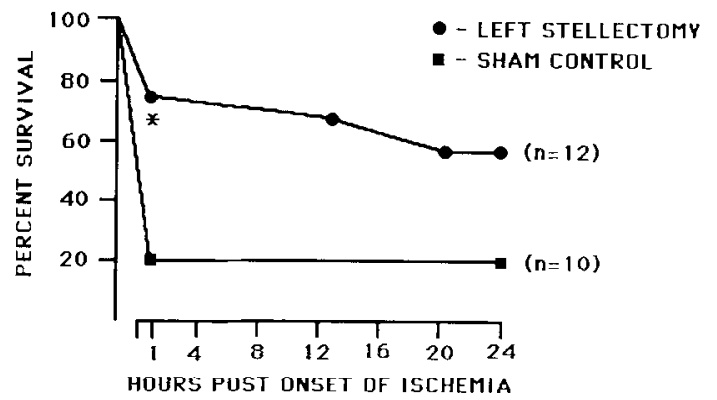

Fig. 4. Twenty-four hour survival in post-infarction dogs with left stellectomy $(n=12)$ or sham denervation $(n=10)$ during acute posterolateral ischemia. ${ }^{*} P<0.05$, at 1 hour after the onset of ischemia.

and $(206 \pm 5$ vs $189 \pm 5 \mathrm{msec} ; P<0.01)$, respectively. In sham controls, similar significant reductions in QTc and paced QT intervals were seen $(314 \pm 10$ vs $287 \pm 9$ msec; $P<0.001)$ and $(211 \pm$ 9 vs $193 \pm 5 \mathrm{msec} ; P<0.05)$. The magnitudes of change in QTc or paced QT intervals were not significantly different between left stellectomy and sham controls. All other electrocardiographic intervals, heart rate, ventricular excitation thresholds and right ventricular outflow tract effective refractory periods were not altered significantly by left stellectomy.

Acute posterolateral ischemia in the setting of a previous anterior myocardial infarction: a model for sudden cardiac death

Five to 7 days after left stellectomy or sham denervation, acute posterolateral ischemia was in-

TABLE 1

Electrocardiographic and electrophysiologic data.

\begin{tabular}{|c|c|c|c|c|c|}
\hline \multirow{3}{*}{$\begin{array}{l}\text { Parameter } \\
\text { Heart rate (beats/min) }\end{array}$} & \multicolumn{2}{|l|}{ Sham control } & \multicolumn{3}{|c|}{ Left stellectomy } \\
\hline & $\overline{\text { PRE }}$ & POST & PRE & \multicolumn{2}{|c|}{ POST } \\
\hline & $122 \pm 6$ & $119 \pm 6$ & $142 \pm 5^{\dagger}$ & 132 & \pm 4 \\
\hline PR interval (msec) & $97 \pm 5$ & $101 \pm 5$ & $95 \pm 4$ & 100 & \pm 3 \\
\hline QRS interval (msec) & $55 \pm 5$ & $54 \pm 2$ & $55 \pm 2$ & 53 & \pm 2 \\
\hline QTC interval $(\mathrm{msec}) /(\mathrm{sec})^{1 / 2}$ & $314 \pm 10$ & $287 \pm 9 * *$ & $292 \pm 9$ & 262 & $\pm 6 * *$ \\
\hline Paced QT interval (msec) & $211 \pm 9$ & $193 \pm 5 *$ & $206 \pm 5$ & 189 & $\pm 5 *$ \\
\hline Ventricular excitation threshold voltage (V) & $2.0 \pm 0.5$ & $2.2 \pm 0.2$ & $1.6 \pm 0.2$ & 2.0 & \pm 0.2 \\
\hline Ventricular refractory period ( $\mathrm{msec}$ ) & $143 \pm 3$ & $140 \pm 4$ & $140 \pm 6$ & 131 & \pm 6 \\
\hline
\end{tabular}

* $P<0.05,{ }^{* *} P<0.01$ pre vs post within the same treatment group.

$\dagger P<0.05$, pre or post between groups. 
duced in conscious post-infarction dogs by electrically-induced thrombosis produced by delivering a continuous $150 \mu \mathrm{A}$ anodal current via the previously positioned electrode in the proximal left circumflex coronary artery (Fig. 1).

Fig. 4 depicts survival for the left stellectomy and sham dogs in the 24-hour period after the onset of posterolateral ischemia. The incidence of ventricular fibrillation occuring within 1 hour after the onset of posterolateral ischemia (i.e. early ventricular fibrillation), was significantly lower after left stellectomy compared to sham-denervated controls (25\% vs $80 \%$, respectively; $P<$ 0.008 ). Two late deaths occurred in the left stellectomy group. The resultant 24-hour survival for the left stellectomy group was 58\% compared to $20 \%$ for sham-denervated controls ( $P=0.072)$.

There was no statistically significant difference in the time to onset of posterolateral ischemia $(245 \pm 45$ vs $188 \pm 44 \mathrm{~min})$, percent increase heart rate at onset of ischemia $(12 \pm 4$ vs $14 \pm 5 \%)$, left circumflex thrombus mass $(14.0 \pm 7.5$ vs $11.4 \pm 4.3$ $\mathrm{mg}$ ) or in the size of previous anterior wall

TABLE 2

Response to ischemia at a site remote from previous myocardial infarction.

\begin{tabular}{|c|c|c|c|}
\hline Parameter & $\begin{array}{l}\text { Left } \\
\text { stellectomy } \\
(n=12)\end{array}$ & $\begin{array}{l}\text { Sham } \\
\text { control } \\
(n=10)\end{array}$ & \\
\hline \multicolumn{4}{|l|}{ Time to ischemia } \\
\hline $\begin{array}{l}\text { Increase in heart } \\
\text { rate at time } \\
\text { of onset }\end{array}$ & & & \\
\hline of ischemia & $12 \pm 4 \%$ & $14 \pm 5 \%$ & NS \\
\hline \multicolumn{4}{|l|}{$\begin{array}{l}\text { Incidence of } \\
\text { "sudden" }\end{array}$} \\
\hline ischemic VF * & $3 / 12(25 \%)$ & $8 / 10(80 \%)$ & $P<0.05$ \\
\hline 24-hour mortality & $5 / 12(42 \%)$ & $8 / 10(80 \%)$ & $P=0.072$ \\
\hline \multicolumn{4}{|c|}{ Infarct size ( $\%$ of left ventricle) } \\
\hline Anterior wall & $27.9 \pm 2.4 \%$ & $28.4 \pm 1.7 \%$ & NS \\
\hline $\begin{array}{l}\text { Posterolateral } \\
\text { wall }\end{array}$ & $\begin{array}{l}26.9 \pm 4.8 \% \\
(n=9)\end{array}$ & $\begin{array}{l}31.8 \pm 9 \% \\
(n=2)\end{array}$ & \\
\hline $\begin{array}{l}\text { Thrombus mass } \\
\text { (mg) }\end{array}$ & $14.0 \pm 7.5$ & $11.4 \pm 4.3$ & NS \\
\hline
\end{tabular}

* Incidence of ventricular fibrillation within $60 \mathrm{~min}$ of onset of ischemia. myocardial infarct size $(27.9 \pm 2.4$ vs $28.4 \pm 1.7 \%$ of left ventricle) between the left stellectomy group and sham-denervated controls, respectively (Table 2). An accurate assessment of posterolateral infarction size using the TTC staining technique was limited by the duration of posterolateral ischemia. Posterolateral myocardial infarction sizes could not be determined in animals developing "early" ventricular fibrillation in response to acute posterolateral ischemia, due to the lack of time available for the development of histochemical or morphological evidence of cell death and tissue necrosis in the ischemic area $[15,16]$. Eight sham controls and 3 left stellectomy dogs had early ventricular fibrillation with no detectable posterolateral infarction because the duration of ischemia was less than 1 hour.

\section{Comparison of survivors versus nonsurvivors}

Considering both treatment groups together, post-infarction dogs not surviving acute posterolateral ischemia had significantly longer QTc intervals than survivors ( $281 \pm 8$ vs $262 \pm 7 \mathrm{msec}$; $P<0.05$ ). Heart rate during acute posterolateral ischemia tended to be higher in nonsurvivors than in survivors $(152 \pm 7$ vs $119 \pm 8$ beats $/ \mathrm{min} ; P<$ 0.01 ). Ventricular refractoriness, threshold voltage, and other electrocardiographic intervals did not differ significantly between survivors and nonsurvivors.

In post-infarction dogs with left stellectomy, there was no significant difference in resting heart rate, electrocardiographic intervals or ventricular electrophysiologic parameters in survivors compared to nonsurvivors.

\section{Evidence for lack of regional myocardial denerva- tion after subacute left stellectomy}

Regional myocardial concentrations of norepinephrine were assayed to assess the extent of denervation 1 week after left stellectomy in 5 dogs compared to 5 sham-denervated controls. Fig. 5 presents the results of regional norepinephrine determinations. The mean norepinephrine concentrations of tissue samples harvested from the left ventricle at the level of the papillary muscles was 
$409.70 \pm 9.90 \mathrm{ng} / \mathrm{g}$ after left stellectomy $(n=30$ samples) compared to $428.07 \pm 10.84 \mathrm{ng} / \mathrm{g}$ in sham controls ( $n=30$ samples); $P=$ NS. Small, but significant differences in regional myocardial norepinephrine concentrations between left stellectomy dogs and sham controls did occur in the mid-anteroseptal $(386.54 \pm 42.08$ vs $433.82 \pm 17.71 \mathrm{ng} / \mathrm{g}$; $P<0.05)$ and the mid-posterolateral regions $(451.80 \pm 18.73$ vs $430.97 \pm 7.58 \mathrm{ng} / \mathrm{g} ; \quad P<0.05)$ of the left ventricle. In all other left ventricular segments assayed, norepinephrine concentrations were similar in both treatment groups.

\section{Effectiveness of left stellectomy technique}

In anesthetized, open chest post-infarction dogs, incremental left sympathetic chain electrical stimulation $(1,2,4,8 \mathrm{~Hz} ; 2 \mathrm{msec}$ duration) resulted in progressive augmentations of heart rate, mean arterial pressure and left ventricular force of contraction in the posterolateral region as displayed in Fig. 6. After acute left stellectomy at the site utilized in the present study in conscious dogs, the inotropic, chronotropic and hemodynamic responses to left sympathetic chain stimulation were blunted. Three dogs were challenged also with tyramine to assess the integrity of stored pools of neuronal norepinephrine. Prior to acute left stellectomy, tyramine challenge elicited a $38 \pm 2 \%$ increase in mean arterial pressure, an $18 \pm 1 \%$

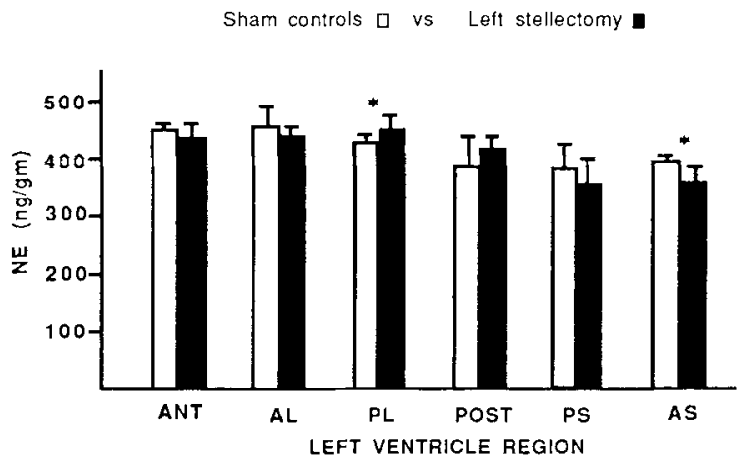

Fig. 5. Regional transmural myocardial norepinephrine (NE) concentrations 1 week after left stellectomy $(n=5)$ or sham denervation $(n=5)$. NE concentrations are expressed as nanograms of NE per gram of tissue. $A N T=$ anterior: $\mathrm{AL}=$ anterolateral; $\quad \mathrm{PL}=$ posterolateral; $\quad \mathrm{POST}=$ posterior; $\quad \mathrm{PS}=$ posteroseptal; AS $=$ Anteroseptal. $* P<0.05$.
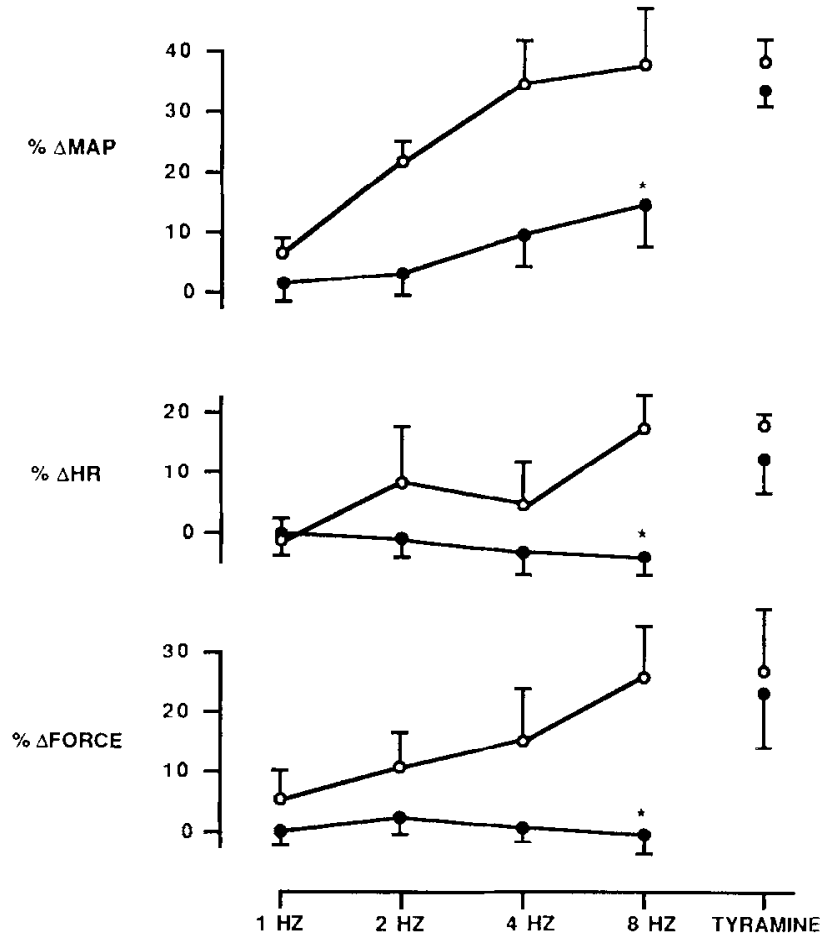

o- PRE-STELLECTOMY - POST-STELLECTOMY

Fig. 6. The effect of incremental left sympathetic chain stimulation and tyramine challenge on mean arterial pressure (MAP), heart rate $(\mathrm{HR})$ and left ventricular force of contraction (FORCE), before $(\varnothing)$ and after $(\bullet)$ acute left stellectomy. Values are expressed as percent change $(\% \Delta)$ from baseline \pm SEM. For 1,2 and $4 \mathrm{~Hz}$ and tyramine, $n=3$. For $\% \Delta \mathrm{MAP}$ and $\% \Delta \mathrm{IIR}$ at $8 \mathrm{IIz}, n-5$. For $\% \Delta$ rORCE at $8 \mathrm{~Hz}, n=4$. ${ }^{*} P<0.05$ PRE vs POST left stellectomy (see text).

increase in heart rate and a $27 \pm 12 \%$ increase in the force of left ventricular contraction. After acute left stellectomy, similar responses in heart rate, mean arterial pressure and force of contraction were observed during tyramine challenge, suggesting that regional myocardial norepinephrine concentrations were not altered by acute left stellectomy.

\section{Discussion}

The results of this investigation confirm and extend previous reports of the antifibrillatory efficacy of the modulation of cardiac sympathetic tone via left stellectomy. This study demonstrates 
a significant reduction in the incidence of "early" ventricular fibrillation (i.e. ventricular fibrillation within 1 hour of the onset of ischemia) in a conscious canine model of recent anterior myocardial infarction in response to acute posterolateral ischemia produced by electrically-induced thrombosis in the left circumflex coronary artery. These results support the findings of Schwartz et al. [10] in a post-infarction canine model using $10 \mathrm{~min}$ balloon occlusion of the left circumflex artery to induce ischemia-related ventricular fibrillation. However, the present investigation demonstrates that the antifibrillatory actions of left stellectomy may not be sustained during a prolonged ischemic challenge. In the present model, "delayed" mortality, which occurred during 1 to 24 hours of posterolateral ischemia, was improved only to a level of borderline statistical significance by left stellectomy compared to control ( $P=0.072$ ). The reason for the discrepancy between early and delayed mortality in our model may be due to differing mechanisms of arrhythmogenesis during prolonged myocardial ischemia [18-20]. A bimodal occurrence of spontaneous ventricular arrhythmias has been described after coronary artery occlusion in dogs. "Early" ventricular arrhythmias tend to occur within the first $20 \mathrm{~min}$ of the onset of myocardial ischemia which are followed by "delayed" arrhythmias which typically develop 4 to 48 hours after the onset of acute coronary artery occlusion. The electrophysiologic mechanisms of initiation and propagation of "early" ventricular arrhythmias may differ from "delayed" ventricular arrhythmias. The results of this study suggest that subacute left stellectomy in a conscious, post-infarction preparation may be more effective against ventricular arrhythmias that occur during the "early" ischemic period.

The experimental model employed in this study is well established in arrhythmia research [13]. In this model, ventricular programmed stimulation identifies a post-infarction preparation with inducible ventricular tachycardia, which is extremely susceptible to the development of ventricular fibrillation during acute posterolateral ischemia produced by electrically induced vasospasm and thrombus formation in the proximal left circumflex coronary artery [12]. Intermittent occlu- sion/reperfusion of the left circumflex coronary artery during intimal electrical stimulation $[12,14]$ results in heterogeneity in recovery of excitability, conduction delay and conduction block in the ischemic bed which predisposes to the development of re-entry and resultant ventricular tachycardia/ventricular fibrillation [21-23].

Interestingly, the time period between the destruction of the left stellate ganglion and the posterolateral ischemic challenge does not appear to be an important factor in determining the antifibrillatory efficacy of left stellectomy. In the study by Schwartz and Stone [10], left stellectomy was performed 1 month prior to an ischemic challenge, while in the current study, a 5- to 7-day delay existed between left stellectomy (subacute) and the posterolateral ischemic challenge. These data suggest that a reduction in either basal or reflex cardiac sympathetic tone rather than regional denervation per se may be an important mechanism of antifibrillatory action.

To test the hypothesis that reduction in regional cardiac sympathetic tone, rather than regional denervation, is a potential mechanism for the antiarrhythmic actions of left stellectomy, we evaluated whether regional myocardial norepinephrine content was altered by subacute left stellectomy. Myocardial norepinephrine concentration is considered a marker of cardiac sympathetic innervation. Previous studies have shown that total cardiac denervation results in dramatic reductions in norepinephrine concentrations within 1 month [24]. To date, no study has evaluated whether destruction of the left stellate ganglion results in regional myocardial denervation. Assessing for regional denervation is important because post-ganglionic projections from the left stellate ganglion are known to innervate primarily the posterior region of the heart [25]. Rcgional differences in norepinephrine concentrations may be overlooked if only global assessments of myocardial norepinephrine concentrations are determined. Experimental noninfarcted canine preparations were used in this portion of the study because myocardial ischemia and/or infarction also may result in myocardial catecholamine depletion which could interfere with the results of regional denervation [26]. Our data suggest that subacute (5-7 days) 
left stellectomy does not induce substantial regional or global myocardial denervation compared to sham controls. Hettering [27] reported similar findings in cats 10 days after left stellectomy. In this feline model, global left ventricle norepinephrine concentrations were not altered, but tritiated norepinephrine uptake was decreased after left stellectomy, suggesting altered neuronal function without loss of neuronal anatomical integrity. Thus, alterations in sympathetic tone rather than regional denervation per se may be an important mechanism of antiarrhythmic action of subacute left stellectomy.

\section{Electrophysiologic effects of subacute left stellec- tomy}

The results of this investigation demonstrate that subacute left stellectomy does not markedly alter cardiac electrophysiologic parameters in a conscious post-infarction dog. Others have demonstrated prolongation of ventricular refractory periods after left stellectomy in anesthetized, open chest canine models $[9,28]$. In the present investigation. left stellectomy did not prolong the ventricular refractory period compared to control. The differences between our study and previous findings may be related to differences in sympathetic tone in the various experimental models employed. Sympathetic tone is known to be augmented by anesthesia and surgery [29-31]. Betaadrenergic stimulation is known to shorten the ventricular refractory period [32].Thus, left stellectomy may indirectly prolong ventricular refractoriness by blunting the electrophysiologic effects of heightened sympathetic tone which occurs with anesthesia and surgery. In contrast, left stellectomy does not appear to alter ventricular refractoriness in the more physiologic setting in a conscious, unsedated preparation. A similar response to beta-adrenoceptor blockade has been demonstrated [32,33]. Beta-adrenoceptor blocking agents devoid of membrane stabilizing properties do not affect ventricular refractoriness in the resting state. However, beta-blockade may blunt the reduction in ventricular refractoriness that. occurs in the presence of heightened sympathetic tone [33].

The discrepancy between this study and previ- ous studies on the effects of left stellectomy on ventricular refractoriness may be related to electrode positioning. In the present study, refractoriness was measured from an electrode positioned in the intraventricular septum near the right ventricular outflow tract. Other studies have shown left stellectomy-related changes in refractoriness measured from the right ventricular apex [9] or the posterior wall of the left ventricle [28].

Left stellectomy does not appear to alter resting heart rate or electrocardiographic intervals. A significant reduction in the QT interval occurred after left stellectomy but a similar reduction over time was seen in the control population suggesting that the QT reduction was probably a manifestation of recovery from the anterior myocardial infarction rather than any direct effects of stellectomy.

When both left stellectomy and control groups are considered together, preparations that did not survive acute posterolateral ischemia had significantly longer QT intervals and greater degrees of sinus tachycardia at the onset of posterolateral ischemia than did survivors. These data obtained in a conscious canine preparation support previous reports that QT prolongation is a possible risk factor for sudden cardiac death in individuals with a previous anterior myocardial infarction [34]. Furthermore, several investigators have observed a relationship between heightened sympathetic neural activity, as expressed by elevated heart rate, and enhanced susceptibility to develop ventricular arrhythmias during myocardial ischemia $[10,35,36]$.

In summary, subacute left stellectomy reduces the incidence of ventricular fibrillation occurring early after the onset of ischemia in a conscious post-infarction canine model. This antiarrhythmic action of left stellectomy does not appear to be related to a measured electrophysiologic action or to regional myocardial denervation.

\section{References}

1 Lown B, Verrier R, Rabinowitz S. Neural and psychological mechanisms and the problem of sudden cardiac death Am J Cardiol. 1977;39:890-902.

2 Malliani A, Schwartz P, Zanchetti A. Neural mechanisms in life threatening arrhythmias. Am Heart J 1980;100:705715 . 
3 Zipes D, Barber M, Takahashi N, Gilmour F. Influence of the autonomic nervous system on the genesis of cardiac arrhythruids. Pate 1983;6:1210-1220.

4 Schwartz PJ. Brown AM, Malliani A, Zanchetti A, eds. Neural mechanisms in cardiac arrhythmias. New York. Raven Press. 1978:107-139.

5 Verrier RL. Thompson P, Lown B. Ventricular vulnerability during sympathetic stimulation: role of heart rate and blood pressure. Cardiovasc Res 1974;8:602-610.

6 Kliks BR, Burgess MJ, Abildskov JA. Influence of sympathetic tone on ventricular fibrillation threshold during experimental coronary occlusion. Am J Cardiol 1975;26: 45-49.

7 Schwartz PJ, Stone HL, Brown AM. Effecis of unilateral stellate ganglion blockade on the arrhythmias associated with coronary occlusion. Am Heart J 1976;92:589-599.

8 Schwartz PJ. Snebold NG, Brown AM. Effects of unilateral cardiac sympathetic denervation on the ventricular fibrillation threshold. Am J Cardiol 1976:37:1034-1040.

9 Schwartz PJ, Verrier RL, Lown B. Effects of stellectomy and vagotomy on ventricular refractoriness. Circ Res 1977; 40:536-540.

10 Schwartz PJ, Stone HL. Left stellectomy in the prevention of ventricular fibrillation caused by acute myocardial ischemia in conscious dogs with anterior myocardial infarctions. Circulation 1980;62:1256-1265.

11 Schwartz PJ, Billman GE, Stone HL. Autonomic mechanisms in ventricular fibrillation induced by myocardial ischemia during exercise in dogs with healed myocardial infarction. Circulation 1984;69:790-800.

12 Wilber DJ, Lynch JJ, Montgomery D, Lucchesi BR. Postinfarction sudden death. Significance of inducible ventricular tachycardia and infarct size in a conscious canine model. Am Heart J 1985;109:8-18.

13 Lynch JJ, Lucchesi BR. How are animal models best used for the study of antiarrhythmic drugs. In: Hearse DJ, Manning AS, Janse MJ, eds. Life-threatening arrhythmias during ischemia and infarction. New York: Raven Press, $1987 ; 169-196$.

14 Patterson E, Holland K, Eller BT, Lucchesi BR. Ventricular fibrillation resulting from ischemia at a site remote from previous myocardial infarction: a conscious canine model of sudden cardiac death. Am J Cardiol 1982;50:1414-1423.

15 Fishbcin MC, Mccrbaum S, Rit $\mathbf{J}$ ct al. Early phase acute myocardial infarct size quantification: validation of the triphenyl tetrazolium chloride tissue enzyme staining technique. Am Heart J 1981;101:593-600.

16 Vivaldi MT. Kloner RA, Schoen FJ. Triphenyl-tetrazolium staining of irreversible ischemic injury following coronary artery occlusion in rats. Am J Pathol 1985;121:522-530.

17 Goldstein DS, Feverstein G, Izzo JL, Kopin IJ, Keiser HR. Validity and reliability of liquid chromatography with electrochemical detection for measuring plasma levels of norepinephrine and epinephrine in man. Life Sci 1981:28:467-475.

18 Scherlag BJ, El-Sherif N, Hope R, Lazzara R. Characteriza- tion and localization of ventricular arrhythmias resulting from myocardial ischemia and infarction. Circ Res 1974;35:372-383.

19 El-Sherif N, Mehra R, Gough WB, Zeiler RH. Ventricular activation patterns of spontaneous and induced ventricular rhythms in canine one-day old myocardial infarction. Circ Res 1982:51:152-166.

20 Davis J, Glassman R, Wit AL. Method for evaluating the effects of antiarrhythmic drugs on ventricular tachycardias with different electrophysiologic characteristics and different mechanisms in the infarcted canine heart. Am J Cardiol 1982;49:1176-1184.

21 Han J, Moe BK. Nonuniform recovery of excitability in ventricular muscle. Circ Res 1964;14:44-60.

22 Merx W. Yoon MS. Han J. The role of local disparity in conduction and recovery time on ventricular vulnerability to fibrillation. Am Heart J 1977;94:603-610.

23 Janse MJ, Kleber AG. Electrophysiologic changes and ventricular arrhythmias in the early phase of regional myocardial ischemia. Circ Res 1981;49:1069-1081.

24 Pivola DV, O’Brien WJ, Parl WG, Simpson WW. Cardiac catecholamine stores after cardiac sympathectomy, 6-OID and cardiac denervation. Am J Physiol 1981;240:H889H895.

25 Randall WG, Szentivanyi M, Pace JB, Wechsler JS, Kaye MP. Patterns of sympathetic nerve projections onto the canine heart. Circ Res 1968;22:315-323.

26 Mathes P, Gudbjarnanson S. Changes in norepinephrine stores in the canine heart following experimental myocardial infarction. Am Heart I 1971;81;211-219.

27 Hettering $G$. Schiefthaler T. The effect of stellate ganglion excision on the catecholamine content and uptake of ${ }^{3} \mathrm{H}$ norcpincphrine in the heart of the cat. Int J Neuropharmicol 1964;3:65-69.

28 Yanowitz F, Preston JB, Abildskov JA. Functional distribution of right and left stellate innervation into the ventricles: production of neurogenic electrocardiographic changes by unilateral alteration of sympathetic tone. Circ Res 1966;18:416-428.

29 Van Citters RL, Franklin DL, Rushmer RF. Left ventricular dynamics in dogs during anesthesia with alpha-chloralose and sodium pentobarbital. Am J Cardiol 1964; 13:349-354.

30 Peiss CN. Manning JW. Effects of sodium pentobarbital on electrical and reflex activation of the cardiovascular system. Circ Res 1964;14:228-234.

31 Olmstead F, Irvine HP. Hemodynamic changes in dogs caused by sodium pentobarbital anesthesia. Am J Physiol 1966:210:817-826.

32 Inoue $\mathrm{H}$, Zipes DP. Results of sympathetic denervation in the canine heart: supersensitivity that may be arrhythmogenic. Circulation 1987;75:877-887.

33 Amlie JP, Refsum H, Landmark KH. Prolonged ventricular refractoriness and action potential duration after $\beta$-adrenoreceptor blockade in the dog heart in situ. J. Cardiovasc Pharmacol 1982:4:157-162. 
34 Schwartz. PJ, Wolf S. QT internal prolongation as a predictor of sudden death in patients with myocardial infarction. Circulation 1978;57:1074-1077.

35 Fujimoto T, Peter T, Hamamoto H, Mandel WJ. Electrophysiologic observations during the spontaneous initiation of ischemia-induced ventricular fibrillation. Am Heart J 1983; 105:189-209.
36 Hope RR, Williams DO, El-Sherif N, Lazzara R, Scherlag BJ. The efficacy of antiarrhythmic agents during acute myocardial ischenia and the role of heart rate. Circulation 1974:50:507-516. 